\title{
CONDUCTAS ANTISOCIALES EN ADOLESCENTES COMO PRECEDENTE DEL TRASTORNO DE PERSONALIDAD ANTISOCIAL EN ADULTOS PRIVADOS DE SU LIBERTAD
}

\author{
J. Jesús Becerra Ramírez y Evelin Alejandra Galicia Gómez \\ Universidad Nacional Autónoma de México \\ México
}

\begin{abstract}
RESUMEN
La adolescencia es una etapa del desarrollo en el ser humano durante la que se presenta una serie de cambios tanto internos como externos, mismos que son necesarios para alcanzar la madurez física y psicológica; sin embargo, se ha identificado una constante en los casos analizados; es común que desarrollen el interés por conductas antisociales durante esta etapa de su vida, mencionan sentirse atrapados en un medio que les cuestiona todo lo que hacen, catalogándolo como una alteración al orden, son jóvenes que expresan una constante inconformidad con el medio social, sus acciones disruptivas van escalando hasta convertirse en delitos, la gran mayoría comienza destruyendo, robando y lastimando. Para este estudio se implementó un procedimiento no aleatorio de tipo descriptivo, con un marco interpretativo fenomenológico, en pacientes del Centro Varonil de Rehabilitación Psicosocial (CEVAREPSI), mismos que están diagnosticados con trastorno de personalidad antisocial (TPA); mediante entrevistas semiestructuradas se abordó la adversidad que vivieron durante su infancia y adolescencia. Es fundamental identificar si hay otros tipos de trastornos asociados, como el disocial, el negativista desafiante y el de límite de la personalidad, entre otros. El estudio muestra que en la adolescencia de los pacientes se encontraron rasgos que coinciden con trastorno disocial; en la mayoría de los casos éste aparece en la adolescencia, y si no es tratado puede extenderse hasta la edad adulta, donde el problema es denominado TPA.
\end{abstract}

Palabras Clave:

conductas antisociales, trastorno, personalidad, adolescente.

\section{ANTISOCIAL BEHAVIORS IN ADOLESCENTS AS A PRECEDENT FOR ANTISOCIAL PERSONALITY DISORDER IN ADULTS DEPRIVED OF THEIR LIBERTY}

\begin{abstract}
Adolescence is a stage of development in the human being, during which a series of internal and external changes occurs, which are necessary to reach physical and psychological maturity, however, a constant has been identified in the cases analyzed; it is common for them to develop an interest in carrying out antisocial behaviors during this stage of their lives, they mention feeling trapped in a medium that questions everything they do, classifying it as an alteration to order, they are young people who express a constant disagreement with the social environment, their disruptive actions escalate to become crimes, the vast majority begin by destroying, stealing and hurting. For this study, a descriptive non-random procedure was performed, with a phenomenological interpretative framework, in patients of the Centro Varonil de Rehabilitación Psicosocial (CEVAREPSI), who are diagnosed with Antisocial Personality Disorder (APT), through semi-structured interviews, the adversity they experienced during their childhood and adolescence. It is essential to identify if there are other types of associated disorders, such as, for example, the Disocial, the challenging Negativist, the Personality Limit, among others. The study showed that in adolescence of the patients traits were found that coincide with Disocial Disorder, this in most cases appears in adolescence and if it is not treated it can extend to adulthood, where the problem is called TPA.
\end{abstract}

Keywords:

antisocial behaviors, disorder, personality, adolescent.

Bitácora del Artículo:

| Recibido: 24 de noviembre de 2019 | Aceptado: 12 de Junio de 2020 | Publicado en línea: Julio - Diciembre de 2020 | 
Estudios de caso | Conductas antisociales en adolescentes como precedente...| Becerra-Ramírez ẽ Galicia-Gómez

Autoría y Derechos de Propiedad Intelectual

\title{
CONDUCTAS ANTISOCIALES EN ADOLESCENTES COMO PRECEDENTE DEL TRASTORNO DE PERSONALIDAD ANTISOCIAL EN ADULTOS PRIVADOS DE SU LIBERTAD
}

\author{
J. Jesús Becerra Ramírez y Evelin Alejandra Galicia Gómez \\ Universidad Nacional Autónoma de México \\ México
}

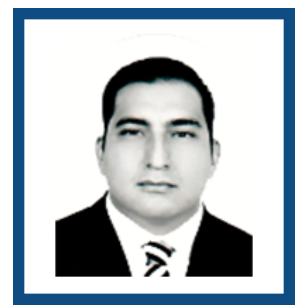

J. Jesús Becerra Ramírez

FES Iztacala UNAM

Correo:berj.jesus@gmail.com

Estudiante de doctorado en Educación; maestro en Diseño de Proyectos Educativos Virtuales por la Universidad Iberoamericana (2012-2014). Licenciado en Pedagogía por la Facultad de Estudios Superiores Acatlán (2004-2007). Actualmente profesor de asignatura en la FES-Iztacala.

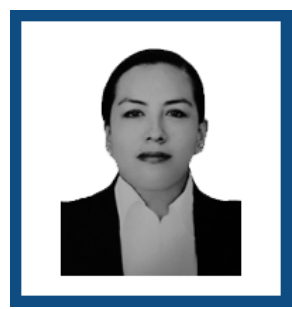

Evelin Alejandra Galicia Gómez

FES Iztacala UNAM

Correo: eagaligom@gmail.com

Maestra en Investigación Criminal y Ciencias Forenses; licenciada en Psicología con mención honorífica, por la Universidad Nacional Autónoma de México, FES Iztacala; como parte de su desarrollo profesional da consulta privada y es profesora del SUAyED en la licenciatura de Psicología desde agosto de 2018

\section{CONTRIBUCIÓN DE LOS AUTORES}

J. Jesús Becerra Ramírez: gestiones administrativas con el personal directivo del Centro Varonil de Rehabilitación Psicosocial (Cevarepsi), revisión literaria, desarrollo de entrevistas, análisis de información y resultados y Evelin Alejandra Galicia Gómez: registro del protocolo ante la Subsecretaría del Sistema Penitenciario, revisión literaria, desarrollo de entrevistas, análisis de información y resultados.

\section{AGRADECIMIENTOS}

El presente artículo no sería posible sin el apoyo del maestro Gumaro Salmerón Nájera y su apreciable equipo de trabajo en el Centro Varonil de Rehabilitación Psicosocial (Cevarepsi), por las facilidades otorgadas y su importante punto de vista acerca de los aspectos abordados. Debo hacer mención especial para la maestra Evelin Alejandra Galicia Gómez por la cordial invitación para colaborar en su proyecto, siempre ofreciendo puntual seguimiento y retroalimentación en todas las actividades efectuadas. También agradezco el trabajo desarrollado por los estudiantes del SUAyED que participaron en los diferentes proyectos implementados de manera conjunta.

\section{DATOS DE FILIACIÓN DE LOS AUTORES}

Facultad de Estudios Superiores Iztacala, UNAM

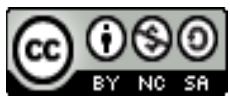

Copyright: (c) 2020 Becerra-Ramírez, J.J. \& Galicia-Gómez, E.A.

Este es un artículo de acceso abierto distribuido bajo los términos de la licencia Creative Commons Reconocimiento-NoComercial 4.0 Internacional, por lo que su contenido gráfico y escrito se puede compartir, copiar y redistribuir total o parcialmente sin necesidad de permiso expreso de sus autores con la única condición de que no se puede usar con fines directamente comerciales y los términos legales de cualquier trabajo derivado deben ser los mismos que se expresan en la presente declaración. La única condición es que se cite la fuente con referencia a la Revista Digital Internacional de Psicología y Ciencia Social y a sus autores. 


\section{TABLA DE CONTENIDO}

INTRODUCCIÓN

434

Características psicológicas del adolescente con conducta

antisocial, 434

\section{MÉTODO}

Identificación del caso, 435

Análisis del motivo de la intervención, 436

Antecedentes del problema, 436

Descripción y análisis del problema, 437

\section{PLAN DE INTERVENCIÓN}

Establecimiento de los objetivos del plan de intervención, 438

Técnicas de evaluación del plan de intervención, 438

EFICACIA DEL PLAN DE INTERVENCIÓN

Aplicación del plan de intervención, 438

Antecedentes familiares, 439

Enfermedades o trastornos, 439

Desarrollo, 439

Pronóstico

Descripción de la fase de seguimiento, 443

Resultados en la fase de seguimiento, 443

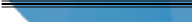




\section{INTRODUCCIÓN}

D urante mucho tiempo el menor con conducta antisocial ha sido tema de discusión e interés de personas y profesionales preocupados por las afectaciones sociales que se pueden ocasionar como consecuencia de dichas acciones; es por ello que diversos profesionales tratan de investigar las causas de su comportamiento con la intensión de identificar las medidas más pertinentes para brindar atención a las personas con ese tipo de conductas.

La adolescencia es una etapa de cambios internos y externos, necesarios para la madurez, tanto física como psicológica, en el ser humano. Los menores que presentan conductas antisociales se sitúan generalmente entre los 11 y los 18 años, por lo que se debe considerar con características biopsíquicas de adolescencia y analizar sus conductas y necesidades de acuerdo con la etapa que están viviendo (Alcántara, 2014).

Es preciso fundamentar los estudios acerca de la conducta antisocial desde los principios de la teoría del desarrollo emocional, pues existen amplias discusiones respecto al tema y varios autores exponen que el trastorno antisocial (TA) se relaciona y origina con las fallas severas vividas por el individuo durante etapas tempranas de su vida, comúnmente provenientes de su familia; el individuo no cubre sus necesidades emocionales, físicas o psíquicas, y no sabe cómo compensarlas, y en consecuencia alberga resentimientos y desarrolla expresiones negativas ante su entorno, como las actividades delictivas o todas las que vayan en contra de la armonía social.

Las conductas antisociales se pueden manifestar en una amplia gama de actividades como desacato de reglas y expectativas sociales, mentiras, acciones agresivas, vandalismo, ausentismo escolar, huidas de casa, consumo de drogas, hurtos, piromanía, etcétera; es decir, son todas las que atentan contra su entorno, incluyendo a personas y propiedades. Cabe destacar que el niño o joven empieza a evolucionar sus conductas antisociales, tornándolas cada vez más gravosas; en este punto es común que el adolescente quede sujeto a los sistemas de justicia penal, pues se pasa de una conducta antisocial a una delictiva; las conductas antisociales vinculadas a la etapa adolescente son el consumo de drogas y otras sustancias adictivas como el alcohol y el tabaco. Durante la adolescencia estas prácticas están asociadas al disfrute del ocio y de las relaciones sociales; los adolescentes están a la búsqueda de la satisfacción personal, deseos de experimentación, desajustes socioafectivos, sobreactivaciones y cuestiones derivadas de intentos de integración en el grupo de iguales (Moral, Rodríguez y Ovejero, 2010).

Al hablar de las conductas antisociales es importante destacar que existen algunos elementos que no se deben pasar por alto, como la edad del sujeto, el sexo, el contexto donde se desarrollan las acciones, así como la magnitud y frecuencia de las mismas; respecto a la edad, hay evidencia de que las actividades antisociales empiezan antes de los 12 años y se prolongan los actos durante la adolescencia y la madurez, aumentando en frecuencia y magnitud (Acevedo, 2011). considerando la etapa de la adolescencia y este conjunto de conductas antisociales, Ceballos (1999) y Aliño-Santiago, López-Esquirol y Navarro-Fernández (2006), identifican este periodo como una etapa de conflicto en la que se produce el tránsito de la niñez a la adultez. El contexto mexicano se caracteriza por tener circunstancias que lo convierten en un entorno vulnerable en la producción y reproducción de la violencia social.

\section{Características psicológicas del adolescente con conducta antisocial}

Es común que las personas con personalidades antisociales sean una población difícil de estudiar, porque no buscan tratamiento por su voluntad y sólo en desterminadas ocasiones discutirán sus problemas con algún médico, psiquiatra o psicólogo (Sue, Wing Sue y Sue, 2010), motivo por el cual es necesario identificar lo más pronto posible los agentes que intervienen en el desarrollo de la conducta antisocial en la edad adulta, y con ello poder confirmar si durante la adolescencia dichas conductas antisociales sirvieron como antesala para el desarrollo de éste o algún otro trastorno asociado.

Una de las características principales es que presenta una alta inconformidad con el medio y rechaza las normas que la sociedad impone, así como la disciplina familiar y todo lo que para el adolescente signifique cumplimiento, esto porque su sentido de responsabilidad es casi nulo, suele tener rasgos muy elevados de agresividad, es manipulador, tiende a proyectar superioridad y autosuficiencia ante las personas que le rodean.

Se pretende identificar las causas por las cuales un menor puede caer en conducta antisocial, destacando que se debe sobre todo a las sucesivas diferenciaciones, divisiones y discriminaciones estructurales de la sociedad; entre ellas podemos indicar los motivos sociopolíticos y socioculturales de la represión; dicha represión es 
por la conservación de valores y mitos en un conjunto social determinado que sirven para la preservación del orden (Van de Valde, 1976). Estas conductas tienen diversas consecuencias, como intoxicaciones, problemas escolares, sexo no planificado, accidentes de tráfico, problemas legales, problemas afectivos, deficiencias de crecimiento y/o desarrollo, fallos orgánicos, consumo de otras sustancias, etcétera (Bartual, Bardisa, López y García-Rodríguez, 2000; Pons y Berjano, 1999).

Por otro lado, toda exclusión social es una agresión a su medio de seguridad, con base en sus carencias emocionales (Ochoa-Alvarez, 1981). La situación de estos adolescentes se explica por medio de los siguientes factores.

- Biológicos. La herencia; ésta influye en la constitución orgánica y psíquica; basado en la teoría de Freud, el sexo está referido a que la mujer moderna delinque igual que el hombre, se considera más la personalidad del individuo, y respecto a la edad se dice que los adolescentes entre 16 y 18 años son los que más delinquen.

- Sociales. Se considera dentro de este factor el ambiente familiar, la escuela, el ambiente urbano, la manera en que el menor tiende a agruparse, etcétera, cuando éstas nos son seleccionadas de modo adecuado y sus contenidos tienen alta carga negativa para el adolescente. La desorganización familiar implica una serie de cambios en la vida de quienes la padecen, perturbando el proceso de socialización, e influirá en la conducta posterior del niño en la conducta desviada y violenta (Núñez, 2010).

Revisando el contexto mexicano, Villatoro-Velázquez et al. (2014), indican que desde 2011 se ha producido un incremento en el consumo de drogas ilegales en la población de 12 a 65 años, siendo los hombres y la población del norte del país quienes son en mayor medida consumidores. Asimismo, consideran que la droga más consumida es la marihuana; la dependencia a las drogas se incrementó de $0.6 \%$ en 2008 a $0.7 \%$ en 2011 (representando a casi 553 mil personas de entre 12 y 65 años), la dependencia al alcohol fue de $6.2 \%$ en 2011, y $21.7 \%$ de la población total de entre 12 y 65 años son fumadores activos.

Otro de los factores que influyen es el fenómeno del pandillerismo, el cual tiene gran presencia en el contexto mexicano, se refiere a "un grupo de personas que forman una alianza basada en necesidades sociales variadas, que participan en actos dañinos para la salud pública y van en contra de los principios y normas de la sociedad" (Alvirde y Del Pazo, 2012: 1); las edades de los miembros de las pandillas oscilan de entre 13 a 21 años, y las causas de ingresos las asocian a cuestiones derivadas de identidad, protección, intimidación, amor propio, excitación de actividades pandilleras, la necesidad de ser aceptados por otros jóvenes, presión de sus compañeros, búsqueda de atención, beneficio financiero y tradición familiar (Rodríguez, 2016).

De aquí surge el interés por elaborar un proyecto que permita sentar las bases para implementar estrategias de atención y prevención al delito en adolescentes ya diagnosticados con el trastorno antisocial y algunos otros asociados. Hoy vivimos en una sociedad muy violenta, y si no se atienden los problemas sociales, cada día empeorarán.

\section{MÉTodo}

\section{Identificación del caso}

Las características comunes entre personalidad y delincuencia han sido objeto de interés para la investigación psicológica y criminológica, pues la mayoría de los delincuentes presentan algunos rasgos de TAP; es conveniente destacar que el individuo típico con dicho trastorno no es leal a nada ni a nadie, rara vez formula una actitud sincera de amistad, afirma respetar la ley y la moral que transgrede de manera constante; además muestra poca culpa, miente y en muchas ocasiones usa a otras personas para sus actos de agresividad, como las personas que participan en la desobediencia civil o violan las convenciones de la sociedad y sus leyes como modo de protesta; no necesariamente tienen personalidades antisociales; por otro lado, si perciben las violaciones de las reglas y normas como actos para un bien mayor, se puede considerar como antisocial (Sue, Wing Sue y Sue, 2010).

Para Marchiori (1975) la Psicología trata de averiguar y conocer qué es lo que induce a un sujeto a delinquir y el significado que tiene esta conducta para él, así como por qué la idea de recibir un castigo no lo atemoriza ni lo hace renunciar a sus conductas criminales. Es por ello que la mayoría de las actividades delincuenciales que perpetran son intencionales y motivadas; ante esto se ha detectado un patrón conductual, pues perciben la sensación de superioridad, lo que los llena de arrogancia hasta el punto en que dejan de protegerse y cometen el delito en circunstancias que se hace inevitable identificarlos como culpables, por lo que reciben el castigo consiguiente; de no ser así insistirán en su comportamiento antisocial hasta recibir la reprimenda familiar, social o penal.

Según Marchiori (1975), cuando se desarrolla el estudio de un determinado delincuente, con una conducta delictiva definida, es necesario recordar que nos 
encontramos con una persona que ha evolucionado en un medio socioeconómico y cultural bien estructurados e identificados, cada uno de ellos con una historia individual, expresando su modo particular de conexión con la realidad. Es por ello que el estudio se hizo en de un centro penitenciario, pues sabemos que el ambiente carcelario está conformado por hostilidad, violencia, sometimiento, abstinencia sexual, de privación afectiva, desesperanza y frustración. Al estar inmerso en un contexto semejante, naturalmente se generan cogniciones, emociones y comportamientos destinados casi sólo a enfrentarlas de modo permanente. Se trata de hacer lo que sea para sobrevivir (Cifuentes y Lodoño, 2011).

\section{Análisis del motivo de la intervención}

La importancia de este estudio estriba en la escasez de trabajos similares dentro del sistema penitenciario, pues este proyecto se efectuó en las instalaciones del Cevarepsi; después de una exhaustiva selección entre diversos reclusos se tomó la decisión de hacer minuciosa investigación con dos casos identificados con el trastorno de la personalidad antisocial, pues encajan a la perfección con el perfil descrito en la literatura; sin embargo, es muy interesante encontrar que los delitos cometidos, y por los cuales están privados de su libertad, son muy diferentes entre sí; por tal motivo se hiso énfasis en las vivencias experimentadas durante su adolescencia, con la intención de compararlas y así identificar características en común. Después se pretenden utilizar los resultados obtenidos para proporcionar un tratamiento específico y/o especializado pensado en las personas con dicho padecimiento, y si es posible aprovechar los hallazgos para fortalecer las estrategias de prevención al delito en adolescentes ya diagnosticados, pues coincidimos con Pitágoras cuando indica: "Educa al niño y no será necesario castigar al hombre". En este caso nos enfocamos en la etapa de la adolescencia, pero los resultados pueden ser aplicables a sujetos de cualquier edad.

El objetivo principal de la investigación es descubrir e identificar las conductas antisociales que presentaron estos internos en su adolescencia, para establecer un programa de prevención de conductas delictivas en adolescentes en conflicto con la ley.

\section{Antecedentes del problema}

El comportamiento antisocial en la infancia y la adolescencia presenta conductas bien identificadas, como trastornos de conducta, resistencia a la autoridad, impulsividad, vandalismo, robo, agresiones físicas y/o psicológicas, maltrato entre iguales, huida de casa, ausentismo escolar y crueldad hacia los animales.
Algunos autores consideran que la familia, la escuela, el medio social y las relaciones son factores o aspectos sociológicos que indican un desfavorecimiento o inferioridad socioeconómica y sociocultural, las cuales propician el desarrollo del TAP, pero esas no son las verdaderas causas, pues a simple vista se puede suponer que existe alguna causa específica de la conducta antisocial en el menor y que se trata de un factor único, pero al estudiarlo más a fondo encontramos múltiples y variados desencadenantes; entonces cuando vemos personas que reúnen varios factores simultáneamente, que además se entrelazan y mezclan, en general los menores comienzan a presentar determinadas conductas; los factores criminógenos de la antisocialidad precoz son susceptibles de cambio; entre ellos podemos encontrar que las conductas antisociales en los adolescentes indican un problema de adaptación, aunque no podemos decir que todo adolescente inadaptado llegue a ser un menor infractor, pero sí se puede hablar de un infractor inadaptado; esta inadaptación se puede considerar de alguna de las siguientes formas.

- Incapacidad para que el individuo pueda adaptar sus conductas a las condiciones del medio en el que se desarrolla.

- Adopción de diversas maneras de conducta que no se adaptan de un modo determinado.

- Inferioridad física o mental, que se origina en su incapacidad para enfrentarse a las exigencias del medio.

En conclusión, podemos decir que la inadaptación social es la incapacidad física, mental y social para integrarse al medio sociocultural aceptado. Los desequilibrios psicológicos son causas de diversas actitudes antisociales; quienes las padecen están en alto riesgo de llegar a delinquir; la falta de entendimiento de los principios éticos, morales y jurídicos que rigen el medio social en que se desenvuelve el adolescente pueden ser también factores predisponentes de la conducta antisocial.

Podemos hablar de otro antecedente; cuando nos referimos a las personalidades psicopáticas, que se presentan cuando los adolescentes llegan a tener problemas con la policía por faltas menores, como la crueldad con animales o conductas vandálicas, dichas conductas les resultan placenteras y se pueden dar por fuerzas instintivas o de malformaciones de carácter. Por otro lado, la farmacodependencia y la drogadicción también son factores que predisponen las conductas antisociales; para obtener estos elementos y satisfacer sus necesidades, el menor comete acciones arriesgadas que lo ponen en peligro.

El contexto sociocultural en que vive el individuo influye en su comportamiento violento; las personas con 
comportamientos delictivos pertenecen a contextos sociales y culturales deprimidos, caracterizados por déficit en servicios públicos, altos niveles de desempleo, baja presencia policial, falta de instalaciones lúdicas, existencia de bandas o grupos organizados para la distribución y consumo de droga, prostitución, pobreza y víctimas de algún acto delictivo (Cifuentes y Lodoño, 2011).

Ochoa-Álvarez (1981) ha encontrado, como dato interesante respecto al problema, que el trastorno antisocial suele ser de mayor presencia en personas sin hogar, destacando que el fenómeno se presenta a menor edad comparado con la norma.

\section{Descripción y análisis del problema}

Para describir y analizar el problema es necesario entender las principales características que encontramos en los sujetos estudiados; es por ello que se revisaron diversos estudios clínicos penitenciarios hechos en México y Argentina; en ellos se indican las características de la personalidad antisocial como las siguientes (Hikal, 2005).

- El individuo con personalidad antisocial es una persona con marcada inestabilidad en todas sus conductas, que proyecta una grave y conflictiva personalidad que se traduce sobre todo en la relación interpersonal agresiva y autodestructiva.

- El antisocial presenta dificultades en el pensamiento, en especial respecto a un pensamiento lógico, debido a que se mueve en un plano muy inmaduro e infantil.

- El juicio está en desconexión con la realidad, y esto posibilita las fantasías y ansiedades persecutorias. Las características están determinadas por la permanente hostilidad y los juicios que hace están en función de dicha hostilidad.

- La comunicación es inestable y predomina un contenido verbal sádico e infantil; tiende a una manipulación y burla de las personas. Por lo general el lenguaje es autoritario.

- En la afectividad el sentimiento de culpa está muy disminuido, con escasa capacidad para experimentar emociones. Frente a los demás el antisocial parece frío y rígido, pero es una protección a sus sentimientos infantiles. Existen fuertes sentimientos internos de inferioridad, de ahí la necesidad del comportamiento agresivo.

Partiendo de que el entorno social en que se desarrollan los individuos tiene mucha influencia en la aparición de los agentes antisociales y su fortalecimiento, fue necesario fundamentar la presente investigación desde el modelo biopsicosocial, pues estas personalidades engloban los tres aspectos del modelo, donde los facto- res sociales desempeñan una función muy importante en el desarrollo de los mismos; la característica principal de la personalidad antisocial es un comportamiento de desprecio y violación a los derechos de los demás, comenzando en la infancia o el principio de la adolescencia, y continuando en la edad adulta; ya en esta última etapa destacan los siguientes indicadores: comportamientos delictivos y/o criminales, abuso de alcohol y/o drogas, rupturas maritales, violencia de género, negligencia en el cuidado de los hijos, conducción temeraria y la agresividad en general.

Debido a sus características personales muchos de los sujetos que sufren de TAP se encuentran recluidos en centros penitenciarios, pues se ven a sí mismos como solitarios, autónomos, fuertes e independientes; a los demás los ven como seres hostiles, desafiantes, vulnerables y con facilidad para ser explotados; sus estrategias principales son las mentiras, agreden, roban, engañan, manipulan, seducen y son exigentes (Caballo, 2004). Los principios morales y activos de la mente son fuertemente pervertidos o depravados; el poder del autogobierno se pierde $y / 0$ deteriora de manera grave,; y el individuo se torna incapaz no de hablar o razonar acerca de cualquier tema que se le propone... sino de conducirse con decencia y propiedad en la vida (Sue, Wing Sue y Sue, 2010).

Han sido diversas las teorías que han intentado explicar los factores que ocasionan la aparición del trastorno antisocial y su desarrollo; algunas se enfocan en el análisis de las diferencias individuales, como los problemas de aprendizaje, conciencia, impulsividad e inteligencia, mientras otras destacan variables externas del individuo, como contexto social, contexto familiar, exposición a la violencia y oportunidades para delinquir (Cifuentes y Lodoño, 2011).

En los casos seleccionados para este estudio encontramos presentes todas las variables mencionadas; por ello es muy importante hacer estos comparativos en casos como los presentados, pues son extremos y están perfectamente identificados.

\section{Plan de intervención}

\section{Descripción del plan intervención}

Se desarrolló un procedimiento no aleatorio donde se utilizó un muestreo por conveniencia (Morales, 2012) dentro de un marco interpretativo fenomenológico, porque se enfoca en la experiencia personal y el mundo; nuestro tipo de investigación fue descriptiva, lo que ayudó a identificar variables que intervienen en el desarro- 
Estudios de caso $\mid$ Conductas antisociales en adolescentes como precedente... | Becerra-Ramírez \& Galicia-Gómez

Ilo de la personalidad antisocial; los pacientes/internos aceptaron de manera voluntaria participar en las entrevistas, proporcionando las evidencias de la intervención en el expediente personal disponible en el Cevarepsi.

\section{Establecimiento de los objetivos del plan de intervención}

Fase 1. Se hizo una entrevista, en la que se preguntó a los internos/pacientes sus antecedentes familiares, con el objetivo de recabar información acerca de enfermedades mentales en la familia, creencias, valores y educación de integrantes de la familia directa y la relación de los pacientes con ellos.

Fase 2. En entrevista se abordaron temas del desarrollo de los pacientes/internos durante la infancia, abarcando de los cinco a los 11 años, para conocer características de su comportamiento en esos primeros años de vida, la forma en que se relacionaban con otros niños y con las autoridades, así como la identificación de alguna enfermedad o padecimiento que les impidiera continuar sus estudios o afectara su desarrollo psicológico o social.

Fase 3. Aplicación de la tercera entrevista a profundidad; en ella se abordaron temas respecto al desarrollo de los pacientes/internos durante la etapa de adolescencia, abarcando de los 12 a los 18 años, para conocer características de su comportamiento, relaciones con otros jóvenes, con autoridades, detección de alguna enfermedad o padecimiento que les impidiera continuar sus estudios o afectara su desarrollo psicológico o social, conocer la edad en que comenzó el consumo de alcohol o drogas, sus hábitos de consumo y el tipo de drogas utilizadas (en caso de ser una respuesta positiva).

Fase 4. Se aplicó la cuarta entrevista; en ella se abordaron temas acerca del desarrollo de los pacientes/ internos durante la etapa adulta, partiendo de los 19 años hasta hoy; se abordaron temas personales, familiares, laborales, sociales, padecimientos médicos, problemas psicológicos, etcétera.

Fase 5. Aplicación de la quinta y última entrevista a profundidad; en ella se habló de manera minuciosa acerca de la causa por la que fueron recluidos en el CEVAREPSI.

Para finalizar con la intervención, se reunió toda la información recopilada de los pacientes/internos y se procedió a efectuar diversos comparativos, dando prioridad a los agentes específicos que se encontraron en cada uno de ellos.

\section{Técnicas de evaluación del plan de intervención}

Entrevistas semiestructuradas. Éstas se pudieron ajustar a los entrevistados. Su ventaja fue la de adaptarse a los sujetos, con enormes posibilidades para motivar al interlocutor, aclarar términos, identificar ambigüedades y reducir formalismos.

1) Ante la Subsecretaría del Sistema Penitenciario se hizo la solicitud de ingreso a las instalaciones del CEVAREPSI para implementar el presente proyecto.

2) Una vez recibido el oficio de respuesta favorable por parte de la Subsecretaría del Sistema Penitenciario, se permitió el acceso al CEVAREPSI, mediante el Área de Psicología de éste, con la supervisión del maestro Gumaro Salmerón Nájera, responsable del área y encargado de seleccionar a los internos/ pacientes entrevistados (ambos con diagnóstico de trastorno de la personalidad antisocial y que no implican alguna situación biológica).

3) Se hizo la presentación formal de los investigadores ante el personal del CEVAREPSI y se recibió una charla informativa referente a derechos humanos, necesaria para poder trabajar con personas privadas de su libertad, sobre todo por tener una situación de inimputabilidad jurídica, debido a sus capacidades diferentes o enfermedades mentales.

4) Se realizó la presentación formal de los investigadores ante los internos/pacientes seleccionados, momento en el que se les invitó a participar en la investigación, dándoles una breve explicación de los objetivos de ésta, proporcionándoles al mismo tiempo un formato de consentimiento informado en el que se explican las condiciones de su participación en el estudio.

5) Después de cumplir con todos los trámites administrativos y con los consentimientos informados firmados, se registró de manera formal el proyecto de investigación ante la Subsecretaría del Sistema Penitenciario, con lo que fue posible comenzar con las entrevistas.

\section{Eficacia del plan de intervención}

\section{Aplicación del plan de intervención}

\section{Caso 1. Datos del interno/paciente}

Edad: 42 años.

Estado civil: Casado.

Escolaridad: Primaria.

Delito: Robo agravado calificado. 


\section{Antecedentes familiares}

Padre. Actualmente vive; tiene estudios de primaria concluidos; de oficio albañil; padece gastritis severa por abuso de sustancias (tabaquismo y alcoholismo). "Siempre ha sido muy callado, seco, grosero, de carácter violento e injusto" (comentario del paciente).

Madre. Falleció hace 14 años; causa de muerte por infarto fulminante; en vida padeció diabetes durante más de 13 años, así como demencia asociada a la diabetes. Sufrió de violencia intrafamiliar por parte del marido durante todo el matrimonio hasta la muerte.

Hermanos. Actualmente viven; tiene dos hermanas; en orden de nacimiento ocupa el tercer lugar de la familia; reporta que sólo se lleva bien con una de sus hermanas, porque la otra tiene el mismo carácter que el padre.

\section{Enfermedades o trastornos}

Epilepsias: Negadas.

TDAH: Negado.

DX: Trastorno mental y del comportamiento secundario a consumo de múltiples sustancias.

\section{Desarrollo}

Infancia (cinco a 11 años). Desde los cinco años el paciente ya tiene conciencia de que su padre es alcohólico; desde esa edad refiere sentirse impotente por los malos tratos que recibían todos los integrantes de la familia por parte del padre. La madre del paciente siempre estuvo al pendiente de ellos; refiere que ella nunca lo golpeó, pero no lo podía controlar; hablaba con él acerca de su comportamiento y sus malas notas escolares, pero menciona que no era buen estudiante; respecto al padre, recuerda que era muy violento, sobre todo si estaba borracho, y si se enteraba que había hecho algo malo, se enojaba con él y lo golpeaba. Manifiesta que durante su niñez era hiperactivo, rezongón y muy desobediente, esto desde que cursada el jardín de niños; si algún compañero en el kínder le hacía una maldad, lo golpeaba. Recuerda que siempre le gustó observar a las personas y cómo se portaban. Durante su etapa en la primaria fue un mal estudiante; reprobó un año porque no le gustaba estudiar; en esta etapa siguieron las peleas con los compañeros, lo que le causó que en el transcurso de la primaria lo cambiaran de escuela en tres ocasiones; los únicos amigos que tenía eran sus "cuates de la colonia". Reporta haber tenido una maestra que lo molestaba mucho, así que la agredió con un explosivo ("paloma"), motivo por el que lo cambiaron de escuela; ya en la otra escuela tuvo un problema con un compañero y lo agredió picándolo con un lápiz, razón por la cual lo expulsaron de modo definitivo y perdió un año escolar.

Adolescencia (12 a 18 años). Con mucha dificultad logró ingresar a la secundaria, porque no lo querían admitir en ninguna escuela debido a sus problemas de conducta y su mal aprovechamiento; refiere que no le gustaba ir a la escuela, no ponía atención, y empezó a tener muchos problemas porque tenía "muchas novias"; esto le creaba severos conflictos, se escapa de la escuela y en este periodo tuvo una riña muy fuerte; cuando su padre se enteró de ésta lo golpeó con el cinturón y con unos alambres; el paciente refiere que en esos momentos sentía mucha rabia y coraje hacía su padre, y en sus pensamientos pasó la idea de querer verlo muerto; como no siguió estudiando, su papá lo lleva a trabajar con él, y refiere que cuando no trabajaba se la pasaba con sus "cuates"; se cansó de trabajar con su padre y se fue a trabajar con una tía en un puesto de discos en Tepito, y fue cuando tuvo su primer acercamiento a las drogas a la edad de 15 años; comenzó consumiendo cocaína; a partir de ese momento se siguió drogando y vendía aparatos "fayuca"; a su vez comenzó a manejar y a vender drogas al menudeo; pasados los 16 años conoció a una chica que le vendía drogas y que le gustaba mucho, pero el ambiente de ella no le agradaba porque su familia era muy influyente en el barrio de Tepito: después de casarse con ella se volvió parte de la familia y se unió al negocio familiar (manejo y venta de todo tipo de drogas, armas y mercancía robada); el padre de la chica era secuestrador y manejaba casi todo el contrabando del área de Tepito.

Edad adulta (19 años, edad actual). Se mueve en el ambiente del tráfico de drogas, compra y venta de armas de fuego y mercancía robada; refiere que durante esa etapa comienzó a obtener grandes sumas de dinero, hasta que fue detenido y encarcelado a los 20 años.

\section{Situaciones sociales}

Situación económica. El paciente manifiesta que en su casa le daban lo que necesitaba; al dejar de estudiar, su padre lo puso a trabajar y después decidió ir a trabaja con su tía vendiendo discos piratas en el barrio de Tepito; ahí conoció las drogas y comienzó a venderlas; a los 16 años conoció a su actual pareja; al formalizar su relación lo iniciaron en el negocio familiar, continuando con la venta de 
Estudios de caso $\mid$ Conductas antisociales en adolescentes como precedente... | Becerra-Ramírez \& Galicia-Gómez

droga, secuestro y muchas otras actividades ilícitas que implicaban la ganancia de mucho dinero.

Violencia. Siempre que su padre consumía alcohol los golpeaba a todos, pero sobre todo a la madre del paciente; esto le hacía sentir mucha impotencia y mucho enojo hacia su papá; desde temprana edad el paciente muestra ser desafiante hacia las figuras de autoridad; manifiesta que tenía una maestra que lo molestaba y decidió agredirla con una paloma explosiva; después picó a un compañero con un lápiz razón por la cual lo cambiaron de escuela y ocasionó que lo expulsaran.

Socialización. El paciente refiere sólo tener "cuates", y éstos eran los que vivían cerca de su casa; dentro de la escuela no hizo amigos, porque no le caían bien por estudiosos; desde muy joven se interesó en las niñas, y manifiesta que siempre se sintió atraído por ellas; también menciona que él no podía ver que le lastimaran o agredieran a una niña porque eso le enojaba mucho y las defendía.

Situación mental del paciente al momento de cometer el ilícito. El paciente refiere no recordar nada del incidente porque estaba bajo el efecto de estupefacientes, pero su esposa le contó que intentó robar a mano armada una motocicleta a su vecino, pero la víctima también tenía un arma de fuego y se negó a entregar el vehículo; al escuchar la discusión comenzaron a salir amigos y familiares de ambos, lo que dio pie a una balacera entre ambos bandos, resultando una persona muerta y varios lesionados por arma de fuego; hoy su caso permanece en proceso de investigación por el cargo de homicidio, pues las pruebas presentadas no son contundentes.

\section{Caso 2. Datos del interno/paciente}

Edad: 31 años.

Estado civil: Unión libre.

Escolaridad: Carrera técnica en sistemas computacionales. Delito: Robo agravado.

\section{Antecedentes familiares}

Padre. Vivo, de profesión agente de ventas, pensionado, originario de Michoacán, padece Diabetes y es insulodependiente, tiene seis hijas de un primer matrimonio.

Madre: Viva, se dedica al comercio, originaria de Colombia, hija única, padece problemas de cadera, en articulaciones y columna.
Hermanos: Seis medias hermanas, con quienes ya no tiene relación, y una hermana con quien no ha perdido el contacto.

\section{Diagnóstico de enfermedades o trastornos}

Epilepsias: Negativo.

TDAH: Diagnosticado a los ocho años.

DX: Trastorno mental y del comportamiento secundario por consumo de múltiples sustancias.

\section{Desarrollo}

Infancia (cinco a 11 años). Desde niño el paciente refiere que era muy agresivo, siempre peleaba a la menor provocación, no le gustaba que los maestros le dieran órdenes, no estudiaba y de manera constante se iba de pinta.

Adolescencia (12 a 18 años). Durante su etapa en la secundaria sobornaba a los maestros para pasar sus materias, pero cuando un profesor no le aceptó el soborno, el informante le dijo que era un "asalariado" y lo expulsaron de la escuela; para terminar la secundaria su mamá lo inscribió en un sistema abierto, pero el paciente confiesa haberla terminado por medio de sobornos. A los 14 comienzó a tener relaciones sexuales, y refiere que desde ese momento tuvo muchas parejas sexuales, ocasionales en su mayoría; "fui muy noviero y mujeriego". A los 16 años se inició en el consumo de alcohol y drogas; empiezó a conocer y a consumir cuando trabajaba en una carnicería, fumando marihuana, y después la cocaína fumada e inhalada.

Edad adulta (19 años, edad actual). Mantiene un trabajo estable en Televisa, aunque aún se droga; en ese periodo la empresa lo envió a rehabilitación durante un año a Oceánica; al salir decidió vivir en unión libre con su novia, con la cual tiene tres hijas; indica que también tiene un hijo que vive con su mamá en Costa Rica; desconoce si tiene más hijos "regados"; siguió trabajando para Televisa, pero - después de un año de abstinencia- seguía drogándose, motivo por el que perdió su trabajo; después se dedicó a la venta de audífonos para celular, y buscaba tener dinero para surtirse de mercancía o para seguir drogandose. Refiere tener cinco ingresos preventivos al Reclusorio, todos por robo. 


\section{Situaciones sociales}

Situación económica. Gracias a que su papá vivía con ellos y le proporcionaba todo lo que necesitaba, de niño no le faltó nada; cuando sus padres se divorciaron las cosas cambiaron y empezaron a vivir de manera más modesta, ya sin el apoyo del padre. En un tiempo cuando estaba ya viviendo con la madre de sus hijas y no tenía trabajo fijo, se dedicó a robar a un Chedraui para llevarle comida a sus hijas, porque no tenía dinero para surtir su negocio ni para darle de comer a su familia. Los otros robos fueron para comprase drogas.

Violencia. El paciente menciona que cada vez que se metía en problemas graves era golpeado por su mamá con el cinturón; indica que él fue de carácter muy violento y agresivo desde niño, y que golpeaba y peleaba constantemente con quien lo molestara.

Socialización. El paciente refiere que cuando niño no tenía amigos y que las amistades que llegaba tener eran sólo con quienes le podían proporcionar drogas o alcohol. Con las mujeres no tiene problemas para relacionarse, "siempre me fue fácil convencerlas de andar conmigo o hacer lo que yo quería".

Situación mental del paciente al momento de cometer el ilícito. El paciente refiere que se le estaba pasando el efecto de la droga cuando entró al Palacio de Hierro para ir al baño y refrescarse, fue cuando lo agarraron y lo acusaron de robo de unos anillos.

\section{Presentación de resultados}

El objetivo de este trabajo fue descubrir las conductas antisociales que durante la adolescencia Ilevaron al desarrollo de la personalidad antisocial en la edad adulta, situación que derivó en problemas con la ley y motivo por el cual están recluidos en el Centro Varonil de Rehabilitación Psicosocial (Cevarepsi); es necesario indicar que en los dos casos revisados se encontraron conductas latentes, pues en ambos casos el trastorno no se da a partir de la edad adulta, ya sea por alguna enfermedad o daño orgánico; en seguida se describen los factores que detonaron el trastorno.

\section{Psicológicos}

Violencia. Respecto a este factor se encontró que los pacientes se desenvolvieron desde edad temprana en ambientes violentos, ambos víctimas de actos de diferente intensidad de violencia; dichos actos fueron hechos por sus padres, familiares, compañeros de clase y/o maestros, lo que nos lleva a considerar a la violencia como un factor importante; en el caso 1 hay un padre violento, agresivo, golpeador y con posibles tendencias machistas; en el caso 2 la madre era quien golpeaba cada vez que tenía una queja de su hijo; estos individuos no tienen una opinión elevada de su propio carácter, compensando con su autoafirmación "yo" con una insistencia en la masculinidad, que en sus relaciones interpersonales se evidencia una persistente voluntad de dominio.

Ausencia de empatía. Los dos pacientes muestran poca empatía a los sentimientos o emociones de las demás personas; en su momento ambos han expresado su desagrado a lo que pueda sentir la gente; desde niños también mostraron poca empatía con compañeros, y sobre todo a las personas que les representaban una autoridad, como padres, jefes o maestros. Ambos mencionaron ser irresponsables en su cuidado sexual, pues a pesar de saber que algunas de sus parejas estaban infectadas con enfermedades de transmisión sexual, tuvieron relaciones sexuales con ellas sin protección, refiriendo no sentir miedo o no importarles contagiarse.

No tienen remordimiento y muestran deshumanización de las víctimas. Durante las entrevistas los pacientes no mostraron remordimiento alguno; cuando se les preguntó si sentían arrepentimiento o culpa por haber dañado o lastimado a las víctimas de sus delitos, ambos pacientes aseguraron de modo rotundo que "era culpa de ellos, pues ya se lo habían ganado", por lo que no sentían remordimientos.

Manipulación. Los pacientes con TPA son manipuladores y utilizan a los demás para lograr sus objetivos; tienen la característica de ser seductores; desde niños lograron sus cometidos, como cambios de escuela, perpetrar robos, cometer homicidios y violaciones; muchas veces manipularon su entorno y las situaciones que los llevaron a efectuar los ilícitos. Estos pacientes lograron manipular sus ambientes para cometer los delitos que les son imputados; se llega a la conclusión de que han manipulado sus síntomas para dar positivo a determinado padecimiento, con la intención de seguir recluidos en el Cevarepsi, pues les resulta un lugar cómodo al ser uno de los reclusorios menos violentos.

Narcisistas. Estos pacientes tienen una idea de sí mismos que han construido, creyendo que son buenos o mejores que los demás; les cuesta trabajo identificarse con otras personas, por lo cual se aíslan; los pacientes entrevistados han manifestado no tener amistades dentro del centro; sólo tienen conocidos y prefieren estar solos; son poco tolerantes, sobre todo cuando no se tratan temas que ellos están dispuestos a discutir; cuando se trata de lo que necesitan, 
Estudios de caso $\mid$ Conductas antisociales en adolescentes como precedente... | Becerra-Ramírez \& Galicia-Gómez

utilizan a las demás personas; presentan dificultades para sentir emociones como tristeza o necesidad afectiva; en ambos casos se presentan rasgos narcisistas; mencionan siempre hacer mejores cosas que los demás; no se identifican con otras personas.

\section{Sociales}

Hogares caóticos. Los pacientes entrevistados provienen de hogares con dinámicas distintas; el paciente del caso 1 vivió en un hogar con violencia intrafamiliar; ambos pacientes refirieron odiar al padre por la violencia y las agresiones vividas durante el tiempo que vivieron con ellos; ambos padres presentaron problemas con el alcohol; por otro lado, el caso 2 manifiesta haber sido violentado por la madre, pero justifica su comportamiento porque él siempre se portaba mal y ella tenía que hacerlo.

Situación económica (pobreza). En el caso 2, aunque al principio no tenía problemas económicos porque vivía con ambos padres, al momento del divorcio se vieron en una situación moderada, pero no llegaron a situación de pobreza extrema; la necesidad de tener dinero le vino cuando no tenía para consumir drogas, lo cual lo orilló a robar; por esta razón hubo ocasiones en que no tenía dinero para dar de comer a sus hijas; esto lo utiliza cada vez como justificante para efectuar el siguiente robo. En el otro caso la situación económica no se considera factor porque proviene de una familia con ingresos suficientes que les permitían cubrir sus necesidades; hasta que dejaron la escuela se vieron obligados a trabajar; al margen del efecto de las drogas y de la expresión del crimen organizado, se puede deducir que el incremento de la pobreza, así como la corrupción, delincuencia y violencia, son factores predominantes en el desarrollo de trastornos de la conducta.

Conductuales. En ambos casos los pacientes presentan características conductuales similares, como desafío ante las figuras de autoridad, poca socialización, dificultad para mantener relaciones sentimentales, agresividad y violencia sin razón aparente; los pacientes entrevistados muestran este tipo de conductas desde temprana edad (peleas con compañeros de clase, altercados con profesores, agresiones hacia familiares, ruptura de reglas y poca estabilidad para conservar un empleo).

\section{Relevancia social del cambio}

Como se puede ver en ambos casos, aunque los pacientes tienen un nivel de inteligencia adecuado, su rendimiento académico fue bajo porque se negaban a participar e insistían en solucionar sus problemas sin ayuda de los demás; la secuencia más probable sería un inicio con diagnóstico de TDAH (lo cual se presenta en el caso 2), quizá hasta el desarrollo de un trastorno negativista desafiante (TND); éstos pueden ser comórbidos con cualquier trastorno internalizado (ansiedad y/o depresión) con una posible evolución al consumo de sustancias adictivas (Vásquez, Feria, Palacios y De la Peña, 2010), por lo cual se le puede considerar también como un agente en el desarrollo de la personalidad antisocial en casos específicos.

Respecto a la adolescencia de los pacientes, se identificaron aspectos que coinciden con trastorno disocial; dicho trastorno se caracteriza por comportamientos en contra de la sociedad (antisociales) que violan los derechos de otras personas, las normas y reglas adecuadas para la edad; todos estos comportamientos están asociados a una serie de situaciones familiares, sociales y escolares, los cuales pueden desarrollarse en la adolescencia y extenderse hasta la edad adulta, donde el problema se denomina TPA (Vásquez, Feria, Palacios y De la Peña, 2010).

En el DSM-V se indican los criterios que se presentan para el diagnóstico del TD, teniendo en cuenta que deben presentarse tres o más criterios en un periodo que comprende entre los 12 meses o menos; además debe presentar por lo menos un criterio en los últimos seis meses.

\section{Agresión a personas o animales}

- De manera constante fanfarronean, amenazan o intimidan a otros (se identificó en ambos casos).

- Con frecuencia inician peleas físicas (se presentó en ambos casos).

- Han llegado a utilizar armas que pueden causar daño físico grave a otras personas (como palo, bate, ladrillo, botella rota, navaja, pistola, etcétera) (se presentó en ambos casos).

- Muestran crueldad física con las personas (se presentó en ambos casos).

- Manifiestan crueldad física con los animales (se presentó en un caso).

- Roban enfrentándose a la víctima (puede ser un ataque con violencia, arrebatar la bolsa, etcétera) (se presentó en ambos casos).

- Han forzado a alguien a una actividad sexual (se presentó en un caso).

\section{Destrucción de la propiedad}

- De modo deliberado causan incendios con la intención de ocasionar daños graves (no se presentó en ningún caso). 
- De manera deliberada destruyen propiedad de otras personas (como útiles escolares, juguetes, ropa, etcétera) (se presentó en ambos casos).

\section{Fraudulencia o robo}

- Ingresan sin permiso a la casa o el automóvil de otra persona (se presentó en ambos casos).

- Mienten para obtener bienes, favores o para evitar obligaciones (se presentó en ambos casos).

- Roban objetos de valor sin enfrentamientos con la víctima (como robo en tiendas, dinero de los padres o familiares, etcétera) (se presentó en ambos casos).

\section{Violaciones graves de normas}

- Durante la noche permanecen fuera de su casa a pesar de las prohibiciones paternas, iniciando este comportamiento antes de los 13 años (se presentó en ambos casos).

- Se escapan de casa durante la noche por lo menos dos veces; o sólo una vez, y tardan en regresar un largo periodo (se presentó en un caso).

- Hacer novillos (irse de pinta) antes de los 13 años (se presentó en ambos casos).

\section{Pronóstico}

\section{Descripción de la fase de seguimiento}

Una opción que puede considerarse en el tratamiento de los pacientes con TPA es el propuesto por Aaron Beck (López, Rodón, Alfano y Cellerino, 2013), que consiste en los siguientes aspectos.

- Aumentar la colaboración con el paciente.

- Trabajar enfocándose en los problemas.

- Identificación de los pensamientos distorsionados asociados a las conductas desadaptativas.

- Enseñar habilidades de resolución de problemas.

- Disminuir la ira y la impulsividad.

- Aumentar la introspección y la conciencia acerca de las diferentes funciones de sus pautas de conducta.

- Ampliar la base para las atribuciones y valoraciones.

- Toma de construcciones positivas.

\section{Resultados en la fase de seguimiento}

En el caso de los pacientes que están en el Cevarepsi se trabajan todas estas técnicas; es importante destacar que los individuos analizados en este estudio no podrán salir en libertad por la gravedad de los delitos cometidos, lo que representa una limitante muy importante porque, aunque son parte de una población concentrada, la demanda del servicio de psicología no es suficiente para atender de manera adecuada cada caso, porque hay demasiados pacientes/internos y esto no permite proporcionar la atención de modo continuo. Es por ello que una de las propuestas sugeridas para futuras investigaciones sería el crear programas en que se involucren actividades que ayuden al paciente a tener un estado psicológico más estable; algunas de las opciones que se ofrecen a la población del Cevarepsi son el desarrollo de aptitudes artísticas (como manualidades, jardinería, pintura y escritura).

Algunas intervenciones preventivas que han resultado exitosas al ser aplicadas en población estándar, con la intención de evitar el desarrollo de los agentes del TPA, van desde mejorar la capacidad social y la conducta prosocial desde la infancia, involucrando a los padres, compañeros y maestros; por ejemplo, las intervenciones universales han demostrado tener un efecto positivo en los problemas de conducta dentro del salón de clases, reforzando las habilidades sociales en la niñez y estrategias multimodales; incluyendo la participación de los padres, los programas multimodales tienen el propósito de proporcionar de modo simultáneo a los niños y sus padres las habilidades necesarias para alentar de manera efectiva el desarrollo de patrones de conducta prosocial (OMS, 2004).

Las intervenciones selectivas son efectivas en la prevención de problemas de conducta; incluyendo programas prenatales y/o infancia temprana y programas efectuados en el hogar o comunidad, hubo una disminución de factores de riesgo para trastornos de conducta, uso de tabaco durante el embarazo, abuso y abandono de menores, y la disminución de los problemas de conducta durante la adolescencia, incluyendo reducción de la violencia y arrestos; por último, las intervenciones indicadas se dirigen a niños cuyos maestros y/o padres han observado que muestran problemas significativos de conducta; los niños se identifican desde jardín de niños, participan en intervenciones como la capacitación en destrezas sociales, habilidades para solución de problemas, sesiones de juego con compañeros prosociales y tutoría académica, y los padres participan en grupos de capacitación que los preparan para la crianza de los hijos. Estas son algunas de las medidas que se pueden considerar desde temprana edad para prevenir que se desarrollen los agentes que intervienen en el desarrollo del TPA (OMS, 2004). 
Estudios de caso | Conductas antisociales en adolescentes como precedente... | Becerra-Ramírez \& Galicia-Gómez

\section{Referencias}

Acevedo, A. J. (2011). De lo antisocial a asesinos en serie. Apuntes para su discusión, 1a. ed. Plaza y Váldes Editores.

Alcántara, E. (2014). Menores con conducta antisocial, 2a. ed. Porrúa.

Aliño-Santiago. M., López-Esquirol, J., \& Navarro-Fernández, R. (2006). Adolescencia: Aspectos generales y atención a la salud. Revista Cubana de Medicina General Integral, 22(1). Recuperado de http://scielo.sld.cu/scielo.php?script=sci arttext\&pid=S0864-21252006000100009\&lng=es\&tlng=es.

Alvirde, G., \& Del Pazo, J. (2004). El poder de las pandillas. Revista de la Universidad del Valle de Atemajac, 18(50), 19-29.

Bartual, V., Bardisa, M., López, C., \& García-Rodríguez, J. A. (2000). Análisis relacional entre consumo de drogas y conducta delictiva. En J. Fernández, J. Herrero y A. Bravo (comps.), Intervención psicosocial y comunitaria. La promoción de la salud y la calidad de vida. Madrid: Biblioteca Nueva.

Caballo, V. E. (2004) Manual de trastornos de la personalidad. Descripción, evaluación y tratamiento. Madrid: Síntesis.

Ceballos, G. C. (1999). El adolescente y sus retos. La aventura de hacerse mayor. Ediciones Pirámide.

Cifuentes, G. J. J., \& Lodoño, A. N. H. (2011). Perfil cognitivo y psicopatológico asociados a la conducta antisocial. International Journal of Psychological Research, 4(1), 58-69.

Hikal, W. (2005). Criminología psicoanalítica, conductual y del desarrollo. La necesidad de sistematizar el conocimiento criminológico: Las criminologías específicas, 1a. ed. México.

López, A., Rondón, J., Alfano, S., \& Cellerino, C. (2013). Psicoterapia para los trastornos y alteraciones de la personalidad: Guías esquemáticas para profesionales, 1a. ed. Editorial Akadia.

Marchiori, H. (1975 [2018]) Psicología criminal, 8a. reimp. México: Porrúa.

Morales, A. E. (2012). Estadística y probabilidades. Santiago de Chile: Universidad Católica de la Santísima Concepción, Instituto Profesional Virginio Gómez.
Moral, M. V., Rodríguez F. J., \& Ovejero, A. (2010). Correlatos psicosociales del consumo de sustancias.

Núñez, J. (2010). Victimología y violencia criminal. Un enfoque criminológico y psicológico. Colección Bibliográfica de Ciencias Jurídico-Penales.

Ochoa-Álvarez, S. (1981). Factores familiares e individuales caractarísticos de los menores infractores en el Distrito Federal. México: UNAM.

Organización Mundial de la Salud (OMS) (2004). Informe del Dept. de Salud Mental y Abuso de Sustancias; en colaboración con el Centro de Investigación de Prevención de las Universidades de Nijmegen y Maastricht (NLM clasificación: WM 140). Disponible en http://www.who.int/mental health/evidence/Prevention_of_mental_disorders_spanish_ version.pdf.

Pons, J., \& Berjano, E. (1999). El consumo abusivo de alcohol en la adolescencia. Madrid: Ministerio del Interior, Plan Nacional sobre Drogas.

Rodríguez, L. (2016). Conductas antisociales, entorno y autoestima en adolescentes mexicanos. Facultad de Trabajo Social y D. H. de la Universidad Autónoma de Nuevo León.

Sue, D., Wing Sue, D., \& Sue, S. (2010). Psicopatología, comprendiendo la conducta anormal, 9a. ed. Cengage Learning Editores.

Van de Valde, G. (1976). Marginalité Sociale et Justice. Bruselas: Instituto de Sociología.

Vásquez, J., Feria, M., Palacios, L., \& De la Peña, F. (2010) Guía clínica para el trastorno disocial. México: Instituto Nacional de Psiquiatría Ramón de la Fuente Muñiz. Serie: Guías Clínicas para la Atención de Trastornos Mentales. Secretaría de Salud. ISBN: 978-607-460-158-9.

Villatoro-Velázquez, J. A., Mendoza-Meléndez, M. A., MorenoLópez, M., Robles, N. O., Fregoso-Ito, D., Bustos-Gamiño, M., \& Elena, M. (2014). Tendencias del uso de drogas en la Ciudad de México: Encuesta de estudiantes, octubre 2012. Salud mental, 37(5), 423-435. 


\section{Meta-Análisis del Artículo}

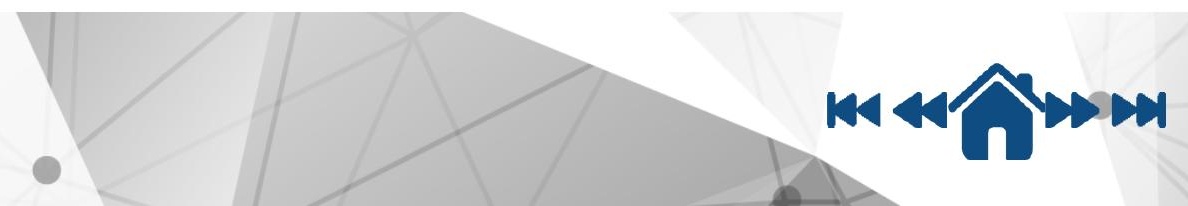




\section{Dimensión Cuantitativa}

\section{Perfil de Evaluación entre pares}
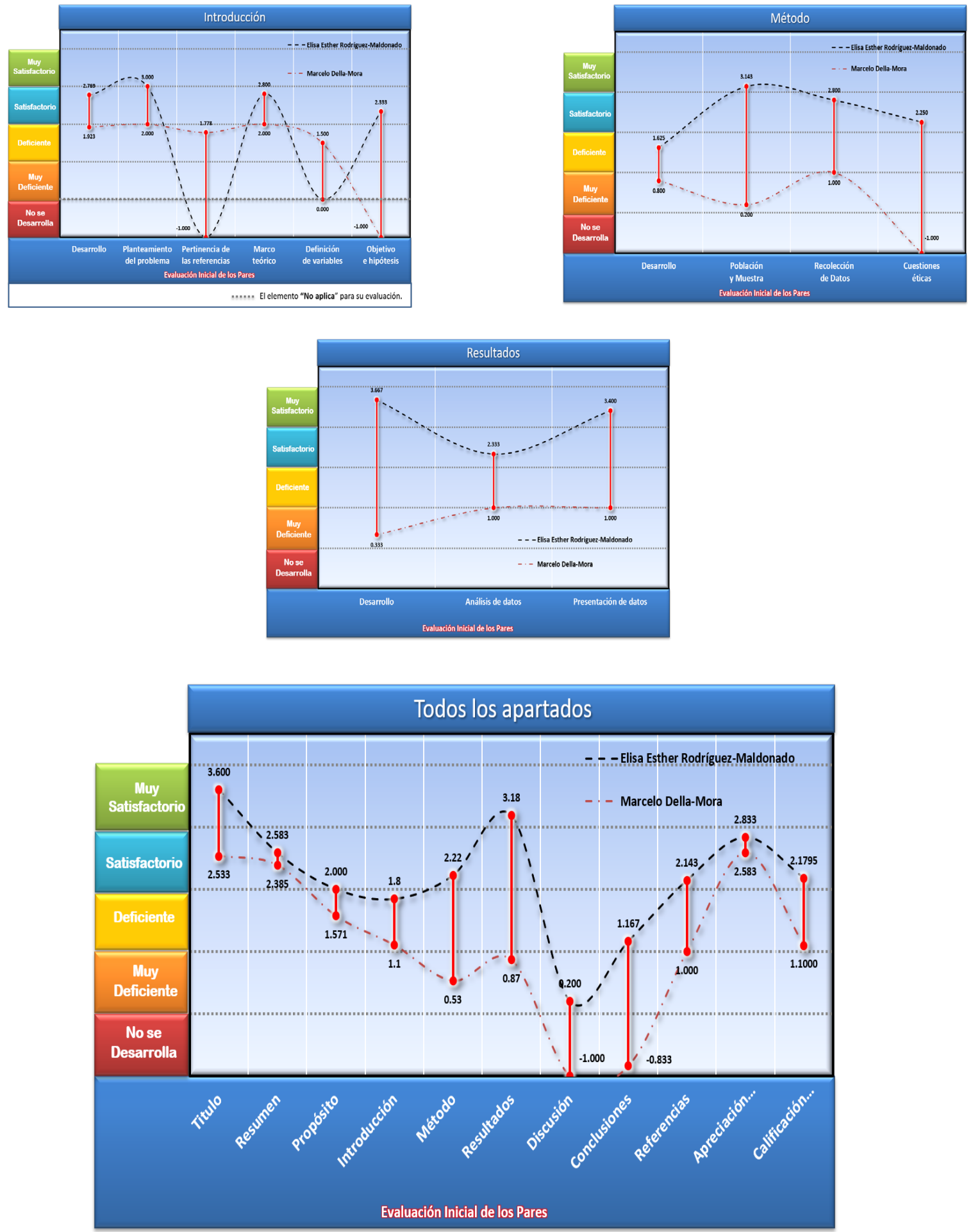


\section{Índice de Concordancia}

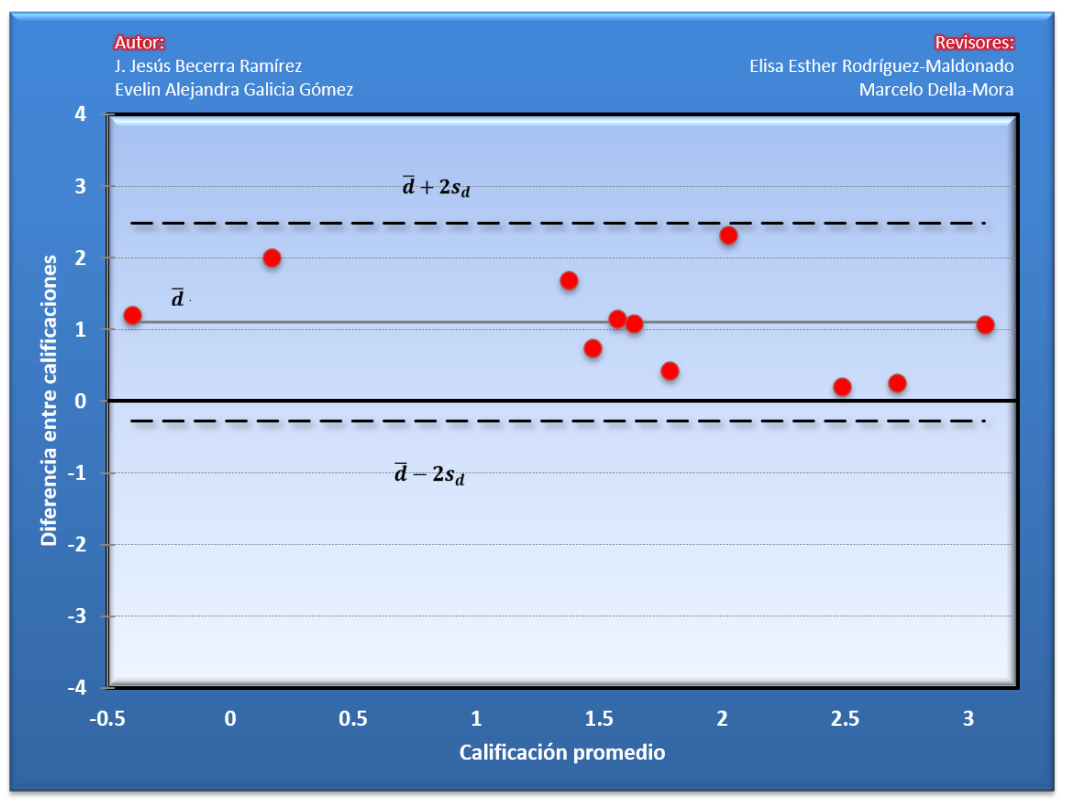

\section{Índice de Acuerdo}

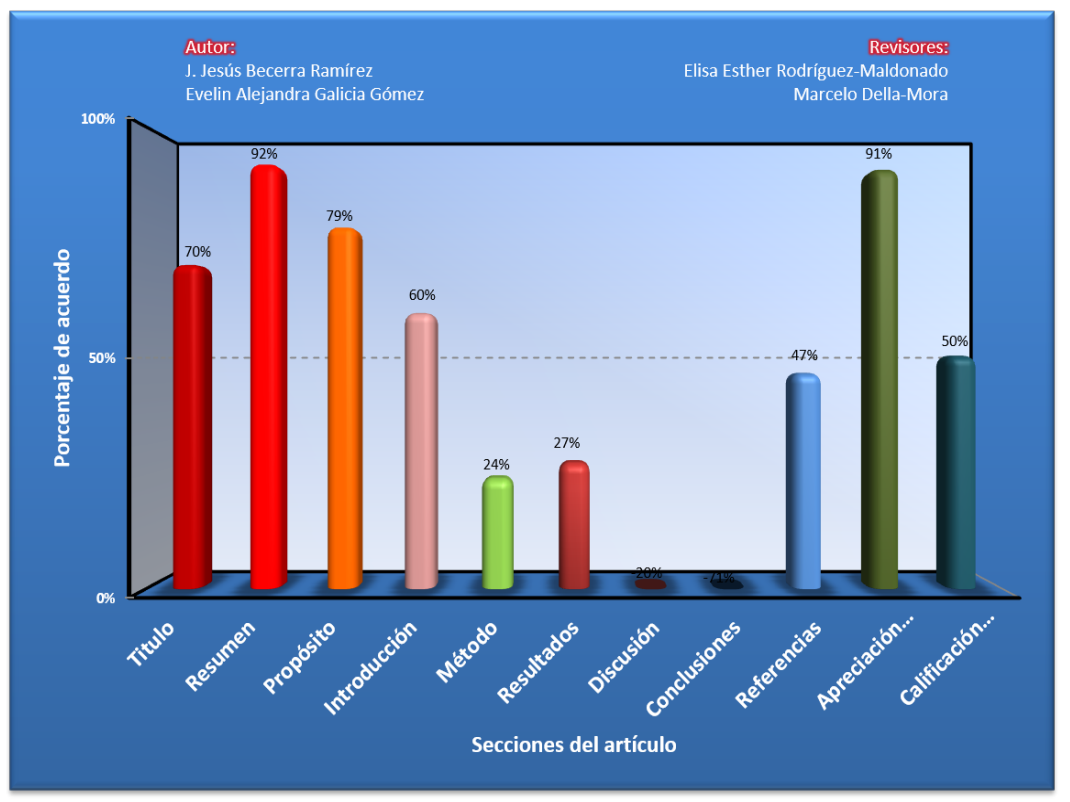




\begin{tabular}{|c|c|}
\hline Revisor 1 & Revisor 2 \\
\hline Elisa Esther Rodríguez-Maldonado & Marcelo Della-Mora \\
\hline \multicolumn{2}{|c|}{ Título/Autoría } \\
\hline $\begin{array}{l}\text { El título tiene más de } 15 \text { palabras. Al escribir en Ado- } \\
\text { lescentes se introduce confusión en cuál es la población } \\
\text { atendida. Se sugiere la siguiente corrección en el títu- } \\
\text { lo para salvar ambos asuntos: Conductas Antisociales } \\
\text { en la Adolescencia como Precedente del Trastorno de } \\
\text { Personalidad Antisocial en Adultos Confinados. En las } \\
\text { palabras clave sería útil incluir "confinados" ( o término } \\
\text { equivalente) sobre la población estudiada. Se utilizan ini- } \\
\text { ciales en el nombre del autor principal - corregir Incluye } \\
\text { grados académicos y posiciones jerárquicas - corregir }\end{array}$ & $\begin{array}{l}\text { El título “Conductas Antisociales en Adolescentes como } \\
\text { Precedente del Trastorno de Personalidad Antisocial en } \\
\text { Adultos Privados de su Libertad" contiene categorías muy } \\
\text { amplias. Se sugiere brindar en el mismo coordenadas es- } \\
\text { pacio-temporales de la investigación y tipo de estudio; } \\
\text { conviene, además aclarar/adelantar que se describirán al- } \\
\text { gunas características de la conducta antisocial juvenil. }\end{array}$ \\
\hline \multicolumn{2}{|l|}{ Resumen } \\
\hline $\begin{array}{l}\text { Mencionar claramente el objetivo de estudio, el diseño } \\
\text { de investigación, el método, los resultados del estudio y } \\
\text { las conclusiones. Utilizar palabras completas, no abre- } \\
\text { viaturas, ni siglas (corregir CE.VA.RE.PSI) Reducir re- } \\
\text { sumen a } 150 \text { palabras (tiene 244) Añadir una palabra } \\
\text { clave que mencione la población bajo estudio (tiene } \\
\text { 4) Clarificar a qué se refiere la última oración del re- } \\
\text { sumen: a la persona? a la población penal? Corregir el } \\
\text { sentido de esa última oración. Para la versión en inglés } \\
\text { del resumen aplicar las mismas correcciones sugeridas } \\
\text { para la versión en español, además de verificar princi- } \\
\text { palmente errores de puntuación. }\end{array}$ & $\begin{array}{l}\text { El resumen no refleja con claridad los pasos del estudio ni } \\
\text { adelanta hallazgos/recomendaciones }\end{array}$ \\
\hline \multicolumn{2}{|c|}{ Próposito del Estudio } \\
\hline $\begin{array}{l}\text { El propósito del estudio guarda relación con el docu- } \\
\text { mento, pero no se concretiza a través del estudio a una } \\
\text { profundidad teórica o práctica suficiente para consti- } \\
\text { tuir un avance en el aporte a las ciencias de la Psicolo- } \\
\text { gía, o a las Ciencias Sociales o Humanísticas. }\end{array}$ & Los objetivos del estudio no están consignados. \\
\hline \multicolumn{2}{|c|}{$\begin{array}{ll} & \text { Introducción } \\
\end{array}$} \\
\hline $\begin{array}{l}\text { Debe desarrollar una revisión de literatura de su tema, } \\
\text { que incluya información de investigaciones similares, } \\
\text { lo que se discute y lo que previamente se sabe sobre } \\
\text { el tema en la comunidad científica, definición de tér- } \\
\text { minos, controversias teóricas, explicación sobre marco } \\
\text { teórico, contraste de teorías, etc. }\end{array}$ & $\begin{array}{l}\text { Los autores anuncian el desarrollo de un marco teóri- } \\
\text { co que luego no realizan. Las fuentes consultadas están } \\
\text { muy desactualizadas. Considerar publicaciones de no } \\
\text { más de diez años. }\end{array}$ \\
\hline
\end{tabular}




\begin{tabular}{|c|c|}
\hline Revisor 1 & Revisor 2 \\
\hline \multicolumn{2}{|c|}{ Método } \\
\hline $\begin{array}{l}\text { Con el método seleccionado no puede responderse la } \\
\text { pregunta de investigación. Debe organizar y separar las } \\
\text { secciones del estudio según el formato estipulado en las } \\
\text { directrices para los autores, ver plantilla. }\end{array}$ & $\begin{array}{l}\text {. Este apartado es bastante confuso. Participantes. Se } \\
\text { sugiere aclarar cuál fue el criterio y el método de selec- } \\
\text { ción de los participantes. Instrumentos. Sería recomen- } \\
\text { dable que agreguen un anexo con las preguntas que uti- } \\
\text { lizaron para recoger los datos. }\end{array}$ \\
\hline \multicolumn{2}{|c|}{ Resultados } \\
\hline $\begin{array}{l}\text { Los resultados pueden presentarse utilizando catego- } \\
\text { rías y criterios clínicos derivados de una revisión de la } \\
\text { literatura científica sobre el tema. Debe responder a la } \\
\text { pregunta de investigación. }\end{array}$ & $\begin{array}{l}\text { Se sugiere agregar un apartado de Discusión a poste- } \\
\text { riori de la presentación de los resultados. Conviene ser } \\
\text { cautos con la generalización de los resultados, por las } \\
\text { características muestrales. }\end{array}$ \\
\hline \multicolumn{2}{|c|}{ Discusión } \\
\hline $\begin{array}{l}\text { Desarrollar una discusión del estudio fundamentada en } \\
\text { una revisión de literatura de estudios similares, compa- } \\
\text { rando los resultados de las categorías estudiadas con in- } \\
\text { vestigaciones previas, señalar hallazgos importantes para } \\
\text { la disciplina científica en la que se enmarca el estudio. }\end{array}$ & $\begin{array}{l}\text { Se sugiere agregar un apartado de Discusión a posteriori } \\
\text { de la presentación de los resultados. Conviene ser cautos } \\
\text { con la generalización de los resultados, por las caracterís- } \\
\text { ticas muestrales. }\end{array}$ \\
\hline \multicolumn{2}{|c|}{ Conclusiones } \\
\hline $\begin{array}{l}\text { Desarrollar el área de conclusiones, conforme a los } \\
\text { aportes científicos del estudio. }\end{array}$ & \\
\hline \multicolumn{2}{|c|}{ Referencias } \\
\hline $\begin{array}{l}\text { Elaborar la revisión de literatura, actualizar las referen- } \\
\text { cias, debe aumentar el número de referencias. }\end{array}$ & $\begin{array}{l}\text { Las citas de las fuentes consultadas, todas, están incom- } \\
\text { pletas y/o están erróneamente referenciadas. Se sugiere } \\
\text { revisar el Manual de Estilo de la American Psychological } \\
\text { Association (APA) para referenciar adecuadamente las } \\
\text { fuentes consultadas. Por otro lado, las fuentes están muy } \\
\text { desactualizadas y son insuficientes; se sugiere ampliar la } \\
\text { lista de fuentes y acotarlas a los últimos diez años. }\end{array}$ \\
\hline
\end{tabular}




\section{Historia del Proceso}

\section{EDITORIAL}

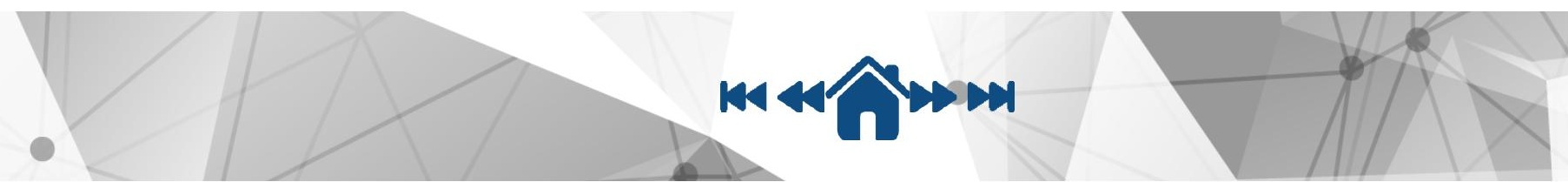

